\title{
Efficacy of preoperative chemoradiotherapy in downstaging rectal cancer and its impact on the long-term outcome
}

\author{
Janaki Gururajachar Manur, Rahil Bharatbhai Patel, Sathish Chandramouli'
}

\begin{abstract}
Introduction: Response to preoperative chemoradiation (PRTCT) for rectal cancer predicts the long-term outcome. Context: Tertiary care hospital. Aims: The aim is to study the factors affecting the response to chemoradiation. Settings and Design: Retrospective. Materials and Methods: Twenty-three patients of rectal cancer undergoing PRTCT followed by surgery and adjuvant chemotherapy were followed up for 20-56 months. Postoperative response, tumor downstaging and nodal downstaging were correlated with the disease status. Results: Tumor downstaging was seen in II (50\%) and nodal downstaging in $12(63.15 \%)$ patients. Nodal downstaging was statistically significant with $P=0.004$. Pathological complete response (PCR) was seen in one patient and partial response (PR) in 17 patients. Thirteen $(72.2 \%$ of patients) were alive and disease free and the negative nodal status correlated with long-term control with $P=0.04$. Conclusion: Most patients of rectal cancer show PR to PRTCT, and the benefit is more for node-positive patients. Nodal PCR is associated with a higher chance of long-term disease control.
\end{abstract}

Key words: Downstaging, long-term outcome, preoperative radiation, rectal cancer

\section{Introduction}

As per ICMR, an increasing trend for rectal cancer is observed only in the southern cities of Bangalore and Chennai among men. ${ }^{[1]}$ Two important developments of mesorectal excision and preoperative chemoradiation (PRTCT) have brought down the local recurrence from $50 \%$ to $<10 \%$ in the last couple of decades. This is probably accompanied by additional possibility of sphincter preservation.

In the compact space of pelvis, rectum is closely related other pelvic structures, and hence, getting an oncological safe margin is technically difficult, and the fact that most part of rectum is devoid of peritoneum, local spread is quite early in the course of disease and hence the role for PRTCT. In a large prospective randomized study by German rectal cancer study group comparing PRTCT with adjuvant chemoradiation for operable rectal cancer, local recurrence was $6 \%$ versus $13 \%$ in favor of PRTCT at 5 years for Stages II and III tumors with $P=0.006 .^{[2]}$ In addition, they observed that there were no increased complications, and sphincter preservation was significantly higher in the RTCT arm. Since then, RTCT has become the standard of care for all patients except $T_{1} T_{2}$ with $\mathrm{N}_{0}$ status. Furthermore, there could be a possibility of sphincter preservation with the shrinkage of tumor.

There are many factors which contribute to the response to PRTCT. Some of them are initial stage of the disease, dose of radiation, timing of surgery, and pathological assessment. An effort was made to correlate these factors with the pathological response and to study their impact on long-term control.

\section{Materials and Methods}

Sixty-eight patients of rectal cancer were treated between January 2013 and December 2015 in the department of radiotherapy of a tertiary care hospital. Forty-five patients were treated with adjuvant or palliative radiation and hence were out of this analysis. All 23 patients underwent complete blood count, renal and liver function tests, and chest X-ray. They also underwent proctosigmoidoscopy, biopsy, and

\begin{tabular}{|l|}
\hline Access this article online \\
\hline Quick Response Code: \\
Q \\
Website: www.sajc.org \\
\hline DOI: 10.4103/sajc.sajc_203_17 \\
\hline
\end{tabular}

Departments of Radiotherapy and 'Surgical Oncology, M. S. Ramaiah Medical College, Bengaluru, Karnataka, India Correspondence to: Prof.Janaki Gururajachar Manur, E-mail:drjanakimg@gmail.com a contrast-enhanced computed tomography (CT) scan of abdomen and pelvis. The tumor extent and nodal status were documented. Tumor was staged as T3 if perirectal stranding was seen and T4 if any other pelvic structures were involved.

All patients underwent PRTCT. A contrast-enhanced CT simulation planning was done. Target for radiation included the gross primary disease with $2 \mathrm{~cm}$ cephalocaudal margin, the entire mesorectum, bilateral internal iliac and presacral nodes from sacral first vertebra to the tip of the coccyx. External iliac nodes were included whenever there was an anterior extension. The combined volume with a $7 \mathrm{~mm}$ all around expansions was the planning target volume to which the dose was prescribed. Radiation was delivered using three-dimensional conformal radiation therapy technique to a dose of $45 \mathrm{~Gy} / 25 \mathrm{Fr} / 5 \mathrm{Fr} /$ week/35 days. An additional dose of $540 \mathrm{cGy} / 3 \mathrm{Fr}$ was given to the gross disease.

All patients received chemotherapy which was either oral capecitabine tablets, $825 \mathrm{mg} / \mathrm{m}^{2}$ given twice a day on all the days or 5 fluorouracil ( $5 \mathrm{FU}$ ) infusion $400 \mathrm{mg} / \mathrm{m}^{2}$ given as intravenous bolus daily for 4 consecutive days during the $1^{\text {st }}$ and the $4^{\text {th }}$ week of radiation with leucovorin $20 \mathrm{mg} / \mathrm{m}^{2}$. Around 4 weeks after chemoradiation, the patients were assessed for surgery, and a repeat CT scan was done.

All patients underwent either anterior resection or abdominoperineal resection with pelvic lymphadenectomy. Histopathological reports were studied in detail, and the T and $\mathrm{N}$ staging was documented. The classification used for assessing the response was documented. The response was correlated with the preoperative staging and was documented as pathological complete response (PCR), partial response (PR), stable disease (SD), or progressive disease. Tumor and nodal response were individually documented. At a follow-up of 20-56 months, the disease status was correlated with the histopathological response.

\section{Statistical methods}

Descriptive statistics was analyzed with SPSS Inc. Released

This is an open access journal, and articles are distributed under the terms of the Creative Commons Attribution-NonCommercial-ShareAlike 4.0 License, which allows others to remix, tweak, and build upon the work non-commercially, as long as appropriate credit is given and the new creations are licensed under the identical terms.

For reprints contact: reprints@medknow.com

How to cite this article: Manur JG, Patel RB, Chandramouli S. Efficacy of preoperative chemoradiotherapy in downstaging rectal cancer and its impact on the long-term outcome. South Asian J Cancer 2019;8:98-101. 
2009. PASW Statistics for Windows, Version 18.0. Chicago: SPSS Inc. McNemar-Bowker test was used for comparison of variables, and Pearson's Chi-squared test was used to test the significance.

\section{Results}

Twenty-three patients were the subjects of this study, and the characteristics of these patients are shown in Table 1. Almost half of our patients were in the fifth and sixth decade, and two-thirds had moderately differentiated carcinoma. None of our patients required colostomy before the start of the treatment.

Tumor response to PRTCT was seen in $82.6 \%$ (19 patients). One patient who had PR the histopathology turned out to be leiomyosarcoma. The number of patients showing PR and PCR was $17(94.5 \%)$ and 1 (5.5\%), respectively. We could not get postoperative $\mathrm{T}$ staging in one and $\mathrm{N}$ status in two patients. Fifteen $(87.3 \%), 2(50 \%)$, and $1(50 \%)$ patients showed response for moderately, well, and poorly differentiated tumors, respectively, and it was not statistically significant $(P=0.411)$. Downstaging with respect to $\mathrm{T}$ and $\mathrm{N}$ status is shown in Table 2. Eleven of $22(50 \%)$ patients showed a downstaging as for as $\mathrm{T}$ stage was considered and eleven of $19(57.9 \%) \mathrm{T}_{3 \text { patient }} \mathrm{s}$ remained $\mathrm{T}_{3}$ post-PRTCT, but it was not statistically significant $(P=0.426)$. Nodal downstaging was seen in $12 / 19$ patients $(63.15 \%)$ which was statistically significant $(P=0.0040)$. Nine of $13 \mathrm{~N}_{1}(69.2 \%)$ patients, whereas 3 of $4 \mathrm{~N}_{2 \text { patient }} \mathrm{s}(75 \%)$ showed downstaging and it was statistically significant $(P=0.004)$.

Response to treatment based on time interval between PRTCT and surgery was not statistically significant $(P=0.426)$. Thirteen patients (68.4\%) showed PR and one patient (5\%) showed PCR when surgery was performed within 12 weeks of completion of PRTCT. Four patients showed PR when surgery was performed after 12 weeks of completion of PRTCT.

Among 18 patients who were alive and disease free, $13(72.22 \%)$ patients had T3, N1 disease at presentation and 17 (94.44\%) had achieved PR and one patient complete response and is shown in Table 3 . Initial response did not show significant correlation with the long-term disease status $(P=0.53)$. Seventeen $(94.4 \%)$ patients with postoperative negative nodal status were alive at a mean follow-up of 38 months with no disease as compared to $1(33.3 \%)$ patient who had positive lymph nodes with $P=0.041$, as shown in Table 4.

\section{Discussion}

This retrospective work is an attempt to document the response to PRTCT in operable rectal cancer and to correlate the response with the long-term outcome. There are many such studies in the western literature; however, there are limited studies from Indian subcontinent wherein the response is correlated with the long-term outcome. It is very important to enhance our understanding, especially since there is an increasing trend so that treatment can be individualized.

In a study by Rohit et al., 126 rectal cancer patients over 5 years were treated with PRTCT followed 4-8 weeks later by surgery. ${ }^{[3]}$ They had given weekly 5 FU whereas we had given during $1^{\text {st }}$ and $5^{\text {th }}$ week of radiation similar to German rectal cancer study. All of our patients underwent South Asian Journal of Cancer Volume 8 •Issue 2 April-June 2019
Table 1: Patient characteristics

\begin{tabular}{lc} 
Total: 23 patients & $n(\%)$ \\
\hline Male:female & $15(65.22): 8(34.78)$ \\
Age (years) & $5(21.73)$ \\
$<40$ & $4(17.39)$ \\
$41-50$ & $6(26.08)$ \\
$51-60$ & $5(21.73)$ \\
$61-70$ & $3(13.04)$ \\
$>70$ & \\
Histopathology & $4(17.39)$ \\
Well differentiated & $17(73.91)$ \\
Moderately differentiated & $2(8.69)$ \\
Poorly differentiated & \\
CT scan based T staging & $19(82.60)$ \\
T3 & $4(17.39)$ \\
T4 & \\
CT scan based N staging & $4(17.39)$ \\
N0 & $15(65.21)$ \\
N1 & $4(17.39)$ \\
N2
\end{tabular}

$\mathrm{CT}=$ Computed tomography

Table 2: $\mathrm{T}$ and $\mathrm{N}$ status before and after preoperative chemoradiation

\begin{tabular}{lcccccc}
\hline \multirow{2}{*}{ Preoperative status } & \multicolumn{5}{c}{ Postoperative status } \\
\cline { 2 - 7 } & T0 & T1 & T2 & T3 & Tx \\
\hline T3 & 1 & 1 & 6 & 11 & 0 \\
T4 & 0 & 0 & 2 & 1 & 1 \\
\hline & N0 & N1 & N2 & NX & \\
\hline N1 & 9 & 4 & 0 & & 2 & \\
N2 & 2 & 1 & 1 & & 0 & \\
\hline
\end{tabular}

surgery whereas $35 \%$ of their patients had either SD or they were not operated at all.

There are different systems of response assessment such as Mandard, Becker, Dworak, or Rodel grading system. ${ }^{[4]}$ We have tried to use the Dworak system. The prognostic value of these is better than those of currently used staging systems (e.g., tumor, node, metastasis staging) for tumors treated by neoadjuvant therapy. We did not have the same system for all patients as the reporting pathologists were different. Hence, the downstaging studied was in terms of $\mathrm{T}$ and $\mathrm{N}$ stage only.

Engineer et al. in their multivariate analysis found that pathological node positivity was associated with a statistically significant $(P=0.001)$ poor survival. ${ }^{[5]}$ In our study, also nodal downstaging was the only significant factor $(P=0.004)$. These patients probably require more aggressive therapy in the form of the further addition of postoperative radiation and/or better chemotherapy. Yeo et al. observed that in about $7 \%-17 \%$ of patients having complete response at primary, residual nodal disease could be present. ${ }^{[6]}$

Ideal timing of surgery is still controversial. Theoretically, maximum reduction is expected to be seen after 20 weeks as observed by Dhadda et al. ${ }^{[7]}$ However, longer the duration, there are chances of tumor regrowth, fibrosis complicating surgery, and delay in instituting adjuvant chemotherapy. Huntington et al. observed that chances of PCR were higher when the interval was beyond 10-12 weeks; however, they also observed that a delay in surgery $(<60$ days vs. $>60$ days increased the chances of positive surgical margin (6.7 vs. $4.8 \%$, 
Table 3: Correlation of response with long-term disease status

\begin{tabular}{lccccc}
\hline Histopathological response (23) patients & \multicolumn{2}{c}{ Disease status (F/U: 20-56 months) } & \multicolumn{2}{c}{ Lost to follow } \\
\cline { 2 - 4 } & NED & Recurrence & Died & & up \\
\hline CR (1) & $1(5.55)$ & 0 & 0 & 0 & 0.531 \\
PR (18) & $14(77.77)$ & $1(5.55)$ & $1(5.55)$ & 2 & Degrees of freedom=1 \\
No response/inadequate information (4) & $3(16.66)$ & 0 & $1(5.55)$ & 0 & \\
\hline
\end{tabular}

$\mathrm{CR}=$ Complete response, $\mathrm{PR}=$ Partial response, $\mathrm{NED}=$ No evidence of disease

Table 4: Correlation of nodal status with disease control at a mean follow-up of 38 months

\begin{tabular}{lcccc}
\hline \multirow{2}{*}{$\begin{array}{l}\text { Nodal status } \\
\text { at surgery }\end{array}$} & \multicolumn{2}{c}{$\begin{array}{c}\text { Disease status at mean } \\
\text { follow-up of } \mathbf{3 8} \text { months }(\%)\end{array}$} & OR $(\mathbf{9 5 \%}$ CI) \\
\cline { 2 - 4 } & Death & Alive & \\
\hline Positive (3) & $2(66.7)$ & $1(33.3)$ & 0.041 & $34(1.4-781.0)$ \\
Negative (18) & $1(5.6)$ & $17(94.4)$ & & \\
Total (21) & $3(14.3)$ & $18(85.7)$ & & \\
\hline
\end{tabular}

$\mathrm{OR}=$ Odds ratio, $\mathrm{CI}=$ Confidence interval

$P=0.009) \cdot{ }^{[8]}$ We did not see any difference between $<12$ weeks and $>12$ weeks, and it is to be noted that we saw only one patient who showed CR and was operated before 12 weeks. Sirohi et al. in their retrospective study on 110 patients found that there were no differences in the pathological response, sphincter preservation, or disease-free survival (DFS) when patients were operated either before or after 8 weeks. ${ }^{[9]}$ Probably, a serial imaging at regular intervals will help to decide the timing of surgery for a given patient.

Most studies have made similar observations except de Campos-Lobato et al. who found that greater PCR $(30.8 \%$ vs. $16.5 \%, P=0.03)$ and lower local recurrence $(1.2 \%$ vs. $10.5 \%$, $P=0.04)$ were associated with surgery beyond 8 weeks. ${ }^{[10]}$ Furthermore, Lyon R90-01 in a randomized trial reported increased tumor downstaging after 8-week interval between preoperative radiotherapy and surgery as compared to $<2$ weeks $(P=0.007) .{ }^{[11]}$ We did not observe significant correlation when comparing disease status at 20-56 months to initial downstaging. This is probably because of small number of patients.

Maas et al. in their study correlated the response with the long-term outcome and at 48 months found that those who had PCR had a DFS of $83.3 \%$ as opposed to $65.6 \%$ and local recurrence of $2.8 \%$ versus $9.7 \%$ when less than PCR was seen with $P=<0.00001 .^{[12]}$ We could not study this aspect as we had only one patient achieving PCR.

There is no consensus as for as adequate lymph node dissection goes after neoadjuvant chemotherapy. Fewer nodes picked up probably means better response. On an average, 3-6 nodes are picked up after chemoradiation compared to 12 nodes if surgery is done upfront. In our study, up to 15 nodes were picked up, and nodal response was the only significant factors $(P=0.004) \cdot{ }^{[13]}$

We observed that complete nodal response to PRTCT had a significant correlation with the outcome at a mean follow-up of 38 months. Similar observations were made by Bujko et al. where nodal status was found to be an independent prognostic factor for DFS and overall survival (OS). ${ }^{[14]}$ Yeo et al. also correlated PCR of the tumor with long-term control and observed that DFS (88.5\% vs. $45.2 \%)$ and OS (94.8\% vs. $72.8 \%)$ were better with $P<0.001$ (for both) in pathological node-negative patients compared to node-positive patients. Nodal response is a 100 better predictor of long-term disease control. ${ }^{[7]}$ An attempt was made not only to study the response to chemoradiation but also to look into its impact on the long-term control. The limitations include small patient number and a retrospective work. Although we saw 68 patients in 3 years, significant number of our patients received adjuvant radiation. This reflects the practice at that time and presently most receive preoperative radiation.

\section{Conclusion}

Most patients of operable rectal cancer show PR to PRTCT. Nodal downstaging is better compared to tumor downstaging, and timing of surgery did not seem to affect the response. Nodal PCR is associated with a higher chance of long-term disease control.

\section{Acknowledgment}

We acknowledge the help rendered by our statistician Mr. Shivaraj.

Financial support and sponsorship

Nil.

\section{Conflicts of interest}

There are no conflicts of interest.

\section{References}

1. Nooyi SC, Murthy NS, Shivananjaiah S, Sreekantaiah P, Mathew A. Trends in rectal cancer incidence - Indian scenario. Asian Pac J Cancer Prev 2011;12:2001-6.

2. Sauer R, Becker H, Hohenberger W, Rödel C, Wittekind C, Fietkau R, et al. Preoperative versus postoperative chemoradiotherapy for rectal cancer. N Engl J Med 2004;351:1731-40.

3. Rohit M, Rakesh K, Thapesh B, Rajesh G, Kim V, Pragyat T, et al. Is neoadjuvantchemoradiation a gold standard for carcinoma rectum? A comprehensive analysis of outcomes and prognostic factors from a tertiary care centre from India. Int J Curr Res 2017;9:47616-9.

4. Svenja T, Rupent L. Tumour regressive grading gastrointestinal carcinoma after neoadjuvant treatment. Front Oncol 2013;3:262.

5. Engineer R, Basu T, Chopra S, Arya S, Patil P, Mehta S, et al. Factors influencing response to neoadjuvant chemoradiation and outcomes in rectal cancer patients: Tertiary Indian cancer hospital experience. J Gastrointest Oncol 2015;6:155-64.

6. Yeo SG, Kim DY, Kim TH, Chang HJ, Oh JH, Park W, et al. Pathologic complete response of primary tumor following preoperative chemoradiotherapy for locally advanced rectal cancer: Long-term outcomes and prognostic significance of pathologic nodal status (KROG 09-01). Ann Surg 2010;252:998-1004.

7. Dhadda AS, Zaitoun AM, Bessell EM. Regression of rectal cancer with radiotherapy with or without concurrent capecitabine - Optimising the timing of surgical resection. Clin Oncol (R Coll Radiol) 2009;21:23-31.

8. Huntington CR, Boselli D, Symanowski J, Hill JS, Crimaldi A, Salo JC, et al. Optimal timing of surgical resection after radiation in locally advanced rectal adenocarcinoma: An analysis of the national cancer database. Ann Surg Oncol 2016;23:877-87.

9. Sirohi B, Barreto SG, Patkar S, Gupta A, DeSouza A, Talole S, et al. Down-staging following neoadjuvant chemo-radiotherapy for locally advanced rectal cancer: Does timing of surgery really matter? Indian J Med Paediatr Oncol 2014;35:263-6.

10. de Campos-Lobato LF, Geisler DP, da Luz Moreira A, Stocchi L, Dietz D, Kalady MF, et al. Neoadjuvant therapy for rectal cancer: The impact of longer interval between chemoradiation and surgery. J Gastrointest Surg 2011; 15:444-50.

11. Francois Y, Nemoz CJ, Baulieux J, Vignal J, Grandjean JP, Partensky C, et al. Influence of the interval between preoperative radiation therapy and South Asian Journal of Cancer Volume 8 • Issue 2 • April-June 2019 
surgery on downstaging and on the rate of sphincter-sparing surgery for rectal cancer: The Lyon R90-01 randomized trial. J Clin Oncol 1999; 17:2396.

12. Maas M, Nelemans PJ, Valentini V, Das P, Rödel C, Kuo LJ, et al. Long-term outcome in patients with a pathological complete response after chemoradiation for rectal cancer: A pooled analysis of individual patient data. Lancet Oncol 2010;11:835-44.

13. Ha YH, Jeong SY, Lim SB, Choi HS, Hong YS, Chang HJ, et al. Influence of preoperative chemoradiotherapy on the number of lymph nodes retrieved in rectal cancer. Ann Surg 2010;252:336-40.

14. Bujko K, Nowacki MP, Nasierowska-Guttmejer A, Michalski W, Bebenek M, Kryj M, et al. Long-term results of a randomized trial comparing preoperative short-course radiotherapy with preoperative conventionally fractionated chemoradiation for rectal cancer. Br J Surg 2006;93:1215-23. 